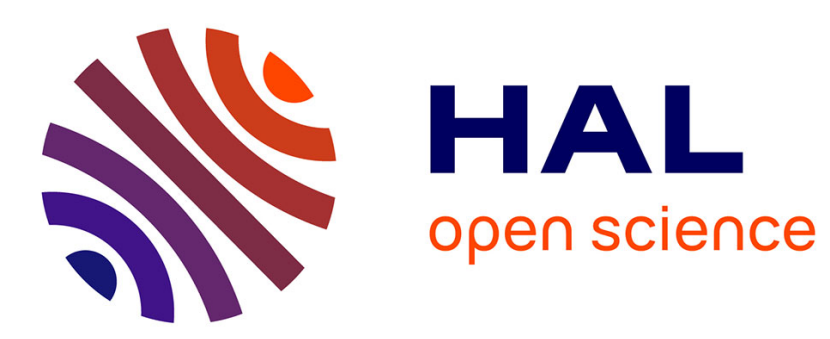

\title{
Multiscale analysis of the structure of homogeneous rotating turbulence
}

\author{
Aurore Naso
}

\section{To cite this version:}

Aurore Naso. Multiscale analysis of the structure of homogeneous rotating turbulence. Physical Review Fluids, 2019, 4 (024609), 10.1103/physrevfluids.4.024609 . hal-02050284

\section{HAL Id: hal-02050284 \\ https://hal.science/hal-02050284}

Submitted on 1 Mar 2019

HAL is a multi-disciplinary open access archive for the deposit and dissemination of scientific research documents, whether they are published or not. The documents may come from teaching and research institutions in France or abroad, or from public or private research centers.
L'archive ouverte pluridisciplinaire HAL, est destinée au dépôt et à la diffusion de documents scientifiques de niveau recherche, publiés ou non, émanant des établissements d'enseignement et de recherche français ou étrangers, des laboratoires publics ou privés. 


\title{
Multiscale analysis of the structure of homogeneous rotating turbulence
}

\author{
Aurore Naso \\ Laboratoire de Mécanique des Fluides et d'Acoustique, CNRS, École Centrale de Lyon and Université Lyon 1, \\ 36 Avenue Guy de Collongue, 69134 Écully Cedex, France
}

(Received 11 September 2018; published 25 February 2019)

\begin{abstract}
The structure of homogeneous rotating turbulence at moderate Reynolds number is investigated by analyzing the instantaneous statistics of the scale-dependent velocity gradient tensor perceived by a set of four fluid elements equally spaced. The relative orientations between dynamical vectors such as vorticity, rate-of-strain eigenframe, and vortex stretching vector, together with their orientations with the rotating frame, are measured by direct numerical simulation at different rotation rates. Measurements are performed in the entire inertial range of scales. The preferential orientation of turbulence with the rotating frame is found to be maximal at the scale of the horizontal large structures of the flow. The relative orientations between dynamical vectors exhibit a continuous and monotonic evolution with scale. Overall, the orientation properties reflect the Gaussianization and two-dimensionalization of turbulence under the effect of rotation. In particular, rotation suppresses some alignment properties valid in isotropic turbulence, which in turn induces a strong decrease of the enstrophy production and strain production rates. These results are found to be valid at all scales.
\end{abstract}

DOI: 10.1103/PhysRevFluids.4.024609

\section{INTRODUCTION}

Dynamical quantities such as enstrophy and strain production, and alignment properties between different vectors such as vorticity and the rate-of-strain eigenframe (the so-called geometrical statistics [1]) are among the most appropriate tools for investigating the physical processes involved in turbulent flows and the structure of turbulence. The information needed to have access to these quantities is contained in the velocity gradient tensor $m_{i j}=\partial_{i} u_{j}$. Many works have been devoted to the modeling of the Lagrangian dynamics of this object, from the restricted Euler dynamics [2,3] to more sophisticated models [4]. The statistical instantaneous properties of $\mathbf{m}$ and of the relations between vorticity and rate of strain were also investigated, in homogeneous and isotropic turbulence (HIT), in a number of experimental and numerical works [5-9].

Another quantity of interest is the perceived velocity gradient tensor $\mathbf{M}$, supported by a set of four fluid elements initially equally spaced [10]. This object allows one to get some insight into the flow topology at any scale and has been the object of various investigations in HIT: Eulerian and Lagrangian measurements in numerical and experimental flows [10-13] or modeling [10,11,14-16] (predictions were also provided for homogeneous shear turbulence [17]).

The dynamics and structure of turbulence are known to be strongly affected by the presence of anisotropy. This symmetry breaking, which occurs, e.g., when the flow is subject to a solid body rotation, induces changes of the fluid properties at all scales. Rotating turbulence has received considerable interest due to its relevance in geophysical and astrophysical flows, but also in many industrial devices (turbomachinery, wind turbines, etc.). Many efforts were made to characterize it, both experimentally [18,19] and numerically [20-22] (see [23] for a recent review). It was found in particular that inverse and direct cascades of energy coexist in rotating turbulence, the former leading to the formation of large-scale vortical structures aligned with the rotation axis [24], and the 
latter to small dissipative structures. Inertial waves [18] can also propagate in these flows and are associated with rapid time scales. According to the resonant wave theory [25-27], two-dimensional three-component slow modes, invariant along the axis of rotation and corresponding to a pure vortex motion, coexist with three-dimensional (3D) fast modes associated with waves [28]. The concentration of energy from the latter to the former can be invoked to explain the formation of vertical large-scale structures in the flow [29-32]. In a different context, the Hopfinger-Zeman scale $[33,34]$ was introduced to quantify the ratio between rotating and inertial effects and is expected to be a threshold between anisotropic (large) and isotropic (small) scales.

We aim here at using the geometrical information provided by the velocity gradient tensor perceived by tetrahedra to quantify the detailed structure of turbulence in a rotating frame, as well as its scale dependence. The Eulerian statistics of $\mathbf{M}$ conditioned on the topology of rotating turbulence were investigated in [35]. Similar measurements of the alignment statistics between vorticity and the rotation vector, related to the cyclone-anticyclone asymmetry arising in rotating turbulence $[18,19,36]$, were then presented in [37].

We extend here these previous investigations by analyzing the relative orientation statistics of other vectors relevant in rotating turbulence: vorticity, rate-of-strain eigenframe, vortex stretching vector, and rotation axis. For this, direct numerical simulations of homogeneous rotating turbulence will be performed. Our results will provide a detailed characterization of the structure of the flow at all scales. At the smallest scales, they will be compared to those presented in [38] in the dissipative range of (inhomogeneous) rotating turbulence. Consequences on the enstrophy production and strain production rates will also be examined.

The paper is organized as follows. We begin in Sec. II by recalling the definitions of the perceived velocity gradient tensor and its properties in HIT and by describing the numerical method. The results are then presented in Sec. III: Orientation statistics of the dynamical quantities with the rotating frame are investigated in Sec. III A, Sec. III B is devoted to the statistical properties of the rate-of-strain tensor, and the implications for the rates of enstrophy production and strain production are examined in Sec. III C. A summary and conclusions are given in Sec. IV.

\section{METHODS AND DEFINITIONS}

\section{A. Standard and perceived velocity gradient tensor}

\section{Definitions}

The usual velocity gradient tensor $m_{i j}=\partial_{i} u_{j}$ allows one to characterize the local topology of the flow [3]. Its symmetric part $\mathbf{s}=\left(\mathbf{m}+\mathbf{m}^{t}\right) / 2$ is the rate-of-strain matrix, while its antisymmetric part is related to vorticity $\boldsymbol{\omega}=\boldsymbol{\nabla} \times \mathbf{u}\left(\omega_{i}=\varepsilon_{i j k} m_{j k}\right)$. Furthermore, incompressibility imposes that $\operatorname{Tr}(\mathbf{m})=\operatorname{Tr}(\mathbf{s})=0$.

The topology of the flow can also be characterized at different scales, in particular in the inertial range. The perceived velocity gradient tensor $\mathbf{M}$, supported by a set of four fluid elements separated by a distance $r_{0}$ from each other $[10,12,13]$, can be used to this aim. Here $\mathbf{M}$ reduces to the standard velocity gradient tensor $\mathbf{m}$ if $r_{0}$ is sufficiently small (in practice, smaller than the Kolmogorov scale $\eta$ ). The time evolution of $\mathbf{M}$ and of the tetrahedron deformation can also be advantageously investigated to address, from a Lagrangian point of view, questions about the fundamental mechanisms of turbulence [10-13,15]. For any set of four fluid elements, the perceived velocity gradient tensor can be defined as follows [10].

If $\mathbf{x}^{a}$ are the positions of the fluid particles and $\mathbf{u}^{a}$ their velocities $(a=1, \ldots, 4)$, then their relative positions and velocities with respect to those of the center of mass of the tetrahedron are $\mathbf{r}^{a}=\mathbf{x}^{a}-\mathbf{x}^{0}$ and $\mathbf{v}^{a}=\mathbf{u}^{a}-\mathbf{u}^{0}$, respectively, where $\mathbf{x}^{0}=\left(\mathbf{x}^{1}+\mathbf{x}^{2}+\mathbf{x}^{3}+\mathbf{x}^{4}\right) / 4$ and $\mathbf{u}^{0}=\left(\mathbf{u}^{1}+\right.$ $\left.\mathbf{u}^{2}+\mathbf{u}^{3}+\mathbf{u}^{4}\right) / 4$. Tensors $\mathbf{g}$ and $\mathbf{Z}$ can then be defined as $g_{i j}=r_{i}^{a} r_{j}^{a}$ and $Z_{i j}=r_{i}^{a} v_{j}^{a}$ (the Einstein summation convention is used throughout the paper). The tensor

$$
\mathbf{M}=\mathbf{g}^{-1} \mathbf{Z}-\operatorname{Tr}\left(\mathbf{g}^{-1} \mathbf{Z}\right) \mathbf{I},
$$


where I denotes the identity tensor, then provides the best-fit approximation of the velocity gradient tensor based on the four points and accounting for incompressibility [13].

Like the true velocity gradient tensor, $\mathbf{M}$ can be naturally decomposed as the sum of its symmetric and antisymmetric parts: $\mathbf{S}=\left(\mathbf{M}+\mathbf{M}^{t}\right) / 2$ is the perceived rate-of-strain matrix and the vector $\boldsymbol{\Omega}$ such that $\Omega_{i}=\varepsilon_{i j k} M_{j k}$ is the perceived vorticity. By virtue of the incompressibility condition, $\operatorname{Tr}(\mathbf{M})=\operatorname{Tr}(\mathbf{S})=0$. Finally, $\mathbf{S}$ and $\boldsymbol{\Omega}$ reduce, respectively, to the real rate-of-strain tensor $\mathbf{s}$ and vorticity vector $\omega$ when $r_{0} \rightarrow 0$.

\section{Properties in homogeneous and isotropic turbulence}

The strain tensor $\mathbf{s}$ being symmetric, it possesses three real eigenvalues that can be ordered as $s_{1} \geqslant s_{2} \geqslant s_{3}$, respectively, associated with the eigenvectors $\hat{\mathbf{s}}_{1}, \hat{\mathbf{s}}_{2}$, and $\hat{\mathbf{s}}_{3}$. The incompressibility condition $\operatorname{Tr}(\mathbf{s})=0$ yields $s_{1}+s_{2}+s_{3}=0$, therefore $s_{1}>0$ and $s_{3}<0$. In recent decades, several experimental and numerical studies were devoted to the characterization of the statistical properties of these eigenvalues and of the orientation of the $\mathbf{s}$ eigenframe with respect to vorticity in 3D HIT.

(a) The intermediate eigenvalue of $\mathbf{s}$ was in particular found to be positive on average: $\left\langle s_{2}\right\rangle>0$ [7-9]. The first important consequence of this result is that the mean strain production $\left\langle-\operatorname{Tr}\left(\mathbf{s}^{3}\right)\right\rangle$ is positive as well $\left[-\operatorname{Tr}\left(\mathbf{s}^{3}\right)=3 s_{1} s_{2} s_{3}\right.$ if the flow is incompressible]; in other words, the rate of strain predominantly self-amplifies.

(b) Investigations of the relative orientation between vorticity and rate of strain showed that vorticity is preferentially collinear with $\hat{\mathbf{s}}_{2}$ and normal to $\hat{\mathbf{s}}_{3}$, whereas no clear preferential alignment can be evidenced between $\omega$ and $\hat{\mathbf{s}}_{1}$ [5-9].

(c) Finally, it was shown that vorticity was preferentially aligned ${ }^{1}$ with the vortex stretching vector $\mathbf{w}$, where $w_{i}=s_{i j} \omega_{j}$ [5-8]. This property, combined with the fact that $\boldsymbol{\omega} \mathbf{S} \boldsymbol{\omega}=\boldsymbol{\omega} \cdot \mathbf{w}=|\boldsymbol{\omega}|$. $|\mathbf{w}| \cdot \cos (\omega, \mathbf{w})$, leads to a preferential vortex stretching (or vorticity production) $\langle\omega \mathbf{s} \omega\rangle>0$, one of the main characteristic trends of 3D HIT.

All these properties drastically distinguish three-dimensional from two-dimensional turbulence, in which (i) vorticity is normal to the $\mathbf{s}$ eigenframe, (ii) $\mathbf{w}=\mathbf{0}$, and (iii) the net rate-of-strain production $\left\langle-\operatorname{Tr}\left(\mathbf{s}^{3}\right)\right\rangle$ and enstrophy production $\langle\omega \mathbf{S} \boldsymbol{\omega}\rangle$ are identically zero.

Similar geometrical statistics of the perceived velocity gradient tensor in 3D HIT were more recently investigated, both experimentally and numerically [13]. The rate of strain and vorticity perceived in the inertial range of scales were found to share many essential properties with their true counterparts $\mathbf{s}$ and $\omega$, a continuity was observed from the dissipative range to the inertial one, and the statistics of $\mathbf{M}$ were shown to tend to Gaussian ones as $r_{0} \rightarrow L$, as expected due to the decorrelation of the velocities at the four points above this scale.

\section{B. Numerical method and parameters}

Incompressible stationary homogeneous rotating turbulence is described by the Navier-Stokes equations written in a rotating frame of reference

$$
\begin{gathered}
\frac{\partial \mathbf{u}}{\partial t}+(\mathbf{u} \cdot \nabla) \mathbf{u}=-\frac{1}{\rho} \nabla p+v \nabla^{2} \mathbf{u}+2 \mathbf{u} \times \boldsymbol{\Omega}_{\mathrm{rot}}+\mathbf{F}, \\
\nabla \cdot \mathbf{u}=0,
\end{gathered}
$$

where $\mathbf{u}(\mathbf{x}, t)$ and $p(\mathbf{x}, t)$ are the velocity and pressure fields, $\rho$ is the fluid density, $v$ is the kinematic viscosity, $\boldsymbol{\Omega}_{\text {rot }}$ is the rotation vector, and $\mathbf{F}(\mathbf{x}, \mathbf{t})$ is a forcing term. In Eq. (2), the pressure field includes the centrifugal force contribution. The results presented in the following section have been obtained by integrating these equations numerically in a cubic domain of size $(2 \pi)^{3}$ with periodic

\footnotetext{
${ }^{1}$ In the present paper we will distinguish the notions of collinearity and alignment: Two vectors $\mathbf{A}$ and $\mathbf{B}$ will be considered as preferentially collinear if $\left\langle\cos ^{2}(\mathbf{A}, \mathbf{B})\right\rangle>1 / 3$ and preferentially aligned if $\langle\mathbf{A} \cdot \mathbf{B}\rangle>0$.
} 
TABLE I. Physical parameters of the runs: $\mathrm{Ro}^{(L)}$ and $\mathrm{Ro}^{(\omega)}$, large-scale and small-scale Rossby numbers, respectively; $\operatorname{Re}_{\lambda}$, Reynolds number based on the Taylor microscale; $\ell_{\mathrm{HZ}}$, Hopfinger-Zeman scale; $\ell_{d}$, scale at which rotation and dissipation equilibrate; $L$, integral scale; and $\eta$, Kolmogorov microscale.

\begin{tabular}{lcccccc}
\hline \hline Run & $\operatorname{Ro}^{(L)}$ & $\operatorname{Ro}^{(\omega)}$ & $\operatorname{Re}_{\lambda}$ & $\ell_{\mathrm{HZ}} / L$ & $\ell_{d} / L$ & $\eta / L$ \\
\hline 1 & $\infty$ & $\infty$ & 190 & $\infty$ & $\infty$ & $3.0 \times 10^{-3}$ \\
2 & 0.087 & 1.7 & 200 & $2.1 \times 10^{-2}$ & $7.1 \times 10^{-3}$ & $4.0 \times 10^{-3}$ \\
3 & 0.051 & 0.73 & 200 & $7.9 \times 10^{-3}$ & $6.3 \times 10^{-3}$ & $5.6 \times 10^{-3}$ \\
4 & 0.027 & 0.24 & 240 & $2.1 \times 10^{-3}$ & $5.3 \times 10^{-3}$ & $8.4 \times 10^{-3}$ \\
\hline \hline
\end{tabular}

boundary conditions. Use was made of a pseudospectral method $[39,40]$ with a resolution of $512^{3}$ collocation points. The advection term was written in a semiconservative way and the viscous one was treated implicitly. Time marching was achieved by a third-order Adams-Bashforth scheme. For the sake of simplicity, the direction of rotation will hereafter be referred to as vertical and the planes perpendicular to it as horizontal.

Forcing was achieved by using the following method, inspired by previous works on HIT [41]: The Fourier modes $\hat{\mathbf{u}}(\mathbf{k}, t)$, for which $|\mathbf{k}| \leqslant 1.5$, obey the Euler equations in the rotating frame, truncated on the same sphere $|\mathbf{k}| \leqslant 1.5$, while the modes for which $|\mathbf{k}|>1.5$ obey the Navier-Stokes equations (2) and (3). The integral scale of the resulting flows is such that $r_{f} / L \in[1.2,1.7]$, where $r_{f}=2 \pi / 1.5$ is the threshold between the truncated Euler system and the Navier-Stokes regime. This forcing method was already used to investigate homogeneous rotating turbulence $[35,37,42]$ and allows one to reach a statistically steady state after a few eddy turnover times starting from a random incompressible velocity field.

The level of anisotropy of the flow can be quantified by the measurement of the largescale and small-scale Rossby numbers, respectively defined as $\operatorname{Ro}^{(L)}=u_{\mathrm{rms}} / 2 L \Omega_{\mathrm{rot}}$ and $\operatorname{Ro}^{(\omega)}=$ $\omega_{\text {rms }} / 2 \Omega_{\text {rot }}$, where $u_{\text {rms }}=\sqrt{\left\langle\mathbf{u}^{2}\right\rangle} / 3, \omega_{\text {rms }}=\sqrt{\left\langle\boldsymbol{\omega}^{2}\right\rangle} / 3$, and $L=2 L_{\text {corr }}$ is the integral scale of the flow, with $L_{\text {corr }}$ defined as the correlation length scale of the Eulerian velocity field $L_{\text {corr }}=$ $\left(\pi / 2 u_{\mathrm{rms}}^{2}\right) \int_{0}^{+\infty} d k E(k) / k[E(k)$ is the energy spectrum]. The other length scales characterizing the flow are the Kolmogorov microscale $\eta$ and the Hopfinger-Zeman scale, defined as $\ell_{\mathrm{HZ}}=$ $\left[\varepsilon /\left(2 \Omega_{\mathrm{rot}}\right)^{3}\right]^{1 / 2}[33,34]$, where $\varepsilon$ is the energy dissipation rate. In rotating turbulence, $\ell_{\mathrm{HZ}}$ quantifies the ratio between inertial and rotation effects and is expected to give a reasonable threshold between anisotropic $\left(r>\ell_{\mathrm{HZ}}\right)$ and isotropic $\left(r<\ell_{\mathrm{HZ}}\right)$ scales, a hypothesis supported by direct numerical simulations $[20,21]$. Other investigations using more refined tools showed that the scale $\ell_{d}=\sqrt{v / 2 \Omega_{\text {rot }}}$, quantifying the ratio between rotation and dissipation, can also be a threshold between isotropic and anisotropic scales in rotating turbulence [42].

Four simulations with approximately the same $\mathrm{Re}_{\lambda}$ but different levels of anisotropy were performed. The values of the main physical parameters for each of them are given in Table I. In run 1 turbulence is isotropic. Rotation increases from run 2 to run 4. In run 2, the Hopfinger-Zeman scale roughly lies in the middle of the inertial range, while in run 4 it is smaller than $\eta$. In this latter run, rotation effects are therefore expected to be dominant over inertial ones at all scales. Typical instantaneous densities of vorticity magnitude in a horizontal plane are displayed in Figs. 1(a)-1(d) for the four runs. The very small structures characteristic of isotropic turbulence [Fig. 1(a)] are replaced by larger ones when rotation is activated [Figs. 1(b)-1(d)]. This feature gets naturally more and more pronounced as rotation is increased, the flow for which $\ell_{\mathrm{HZ}}<\eta$ [run 4 , $\mathrm{Ro}^{(L)}=0.027$, Fig. 1(d)] being qualitatively different from the others. Figure 1(e) shows a snapshot of the normalized vertical component of vorticity in this last run, thereby illustrating the presence of cyclonic and anticyclonic structures in this flow.

As already mentioned, we used sets of fluid elements equally spaced to reconstruct the perceived velocity gradient tensor, a procedure already used in HIT $[10,12,13]$ and in homogeneous rotating turbulence [35,37]. For each value of the Rossby number, several values of $r_{0}$ ranging from less 

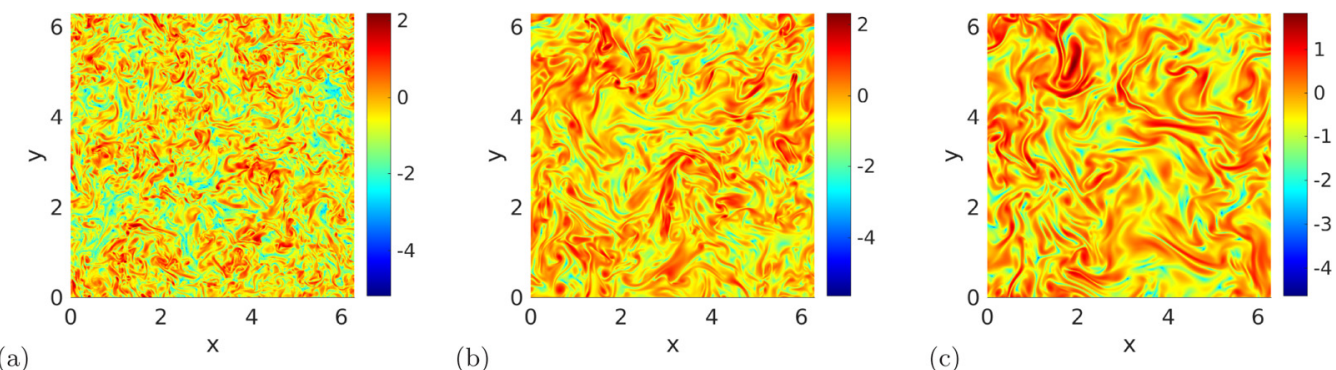

(b)

(c)
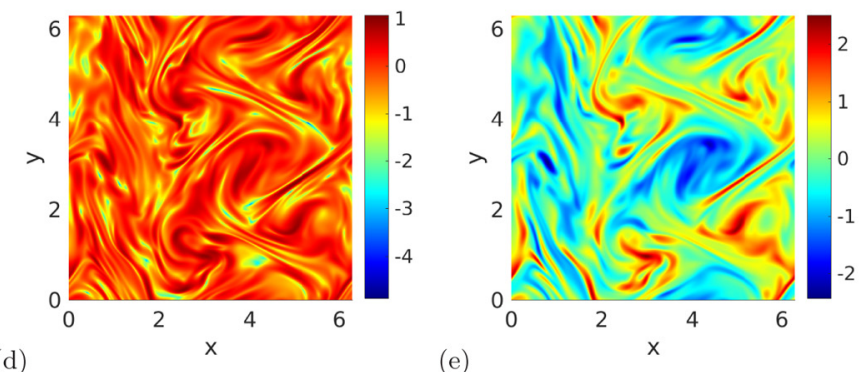

(e)

FIG. 1. Instantaneous visualizations of the flow in horizontal planes for (a)-(d) $\log \left(|\omega| /\left\langle\omega^{2}\right\rangle^{1 / 2}\right)$ and (e) normalized vertical component of vorticity $\boldsymbol{\omega} \cdot\left(\boldsymbol{\Omega}_{\mathrm{rot}} /\left|\boldsymbol{\Omega}_{\mathrm{rot}}\right|\right) /\left\langle\boldsymbol{\omega}^{2}\right\rangle^{1 / 2}$ : (a) isotropic turbulence, (b) Ro ${ }^{(L)}=$ 0.087 , (c) $\mathrm{Ro}^{(L)}=0.051$, and (d) and (e) $\mathrm{Ro}^{(L)}=0.027$. Snapshots (d) and (e) are plotted at the same time and in the same plane.

than or approximately $\eta$ to approximately $L / \sqrt{2}$ were used so as to cover the entire inertial range. In each flow and for each value of $r_{0}$, between $20 \times 10^{6}$ and $40 \times 10^{6}$ randomly oriented tetrads were considered, over a period $\sim(5-6) T_{e}$ (where $T_{e}$ is the large eddy turnover time of the flow) for runs $1-3$ and $\sim 9 T_{e}$ for run 4 . This allowed us to ensure a good convergence of the statistics.

\section{RESULTS}

\section{A. Orientation statistics between dynamical quantities and rotation vector}

Since rotating flows have a preferential direction along the axis of rotation, it is first natural to investigate the orientation between this axis and the vectors characterizing the flow geometry: vorticity, vortex stretching vector, and strain eigenframe. The statistics of alignment between the rotation vector $\boldsymbol{\Omega}_{\text {rot }}$ and perceived vorticity $\boldsymbol{\Omega}\left(r_{0}\right)$ in homogeneous rotating turbulence were investigated by direct numerical simulation in [37]. Both vectors were found to be preferentially antialigned at any scale, a property related to the cyclone-anticyclone asymmetry. The collinearity and antialignment between $\boldsymbol{\Omega}_{\text {rot }}$ and $\boldsymbol{\Omega}\left(r_{0}\right)$ were both found to be maximal at a scale $r_{0} \approx L / 3$.

We now investigate the alignment statistics between the rotation vector $\boldsymbol{\Omega}_{\text {rot }}$ and (i) the vortex stretching vector $\mathbf{W}\left(r_{0}\right)$ (Sec. III A 1) and (ii) the rate-of-strain eigenframe $\left(\hat{\mathbf{S}}_{1}, \hat{\mathbf{S}}_{2}, \hat{\mathbf{S}}_{3}\right)\left(r_{0}\right)$ (Sec. III A 2).

\section{Orientation between vortex stretching vector and rotation axis}

Alignment statistics between the vortex stretching vector $\mathbf{W}\left(r_{0}\right)$, with $W_{i}=S_{i j} \Omega_{j}$, and rotation $\boldsymbol{\Omega}_{\mathrm{rot}}$ are illustrated in Fig. 2(a), which plots the probability distribution functions (PDFs) of the cosine of the angle between the two vectors for the smallest $r_{0}\left(r_{0} \lesssim \eta\right)$ and the three rotating flows simulated. For the smallest Rossby number, both vectors are clearly preferentially normal to each other, which means that the vortex stretching vector preferentially lies in the horizontal plane. Given the fact that vorticity is, in a statistical sense, rather vertical, vortex stretching is therefore expected 

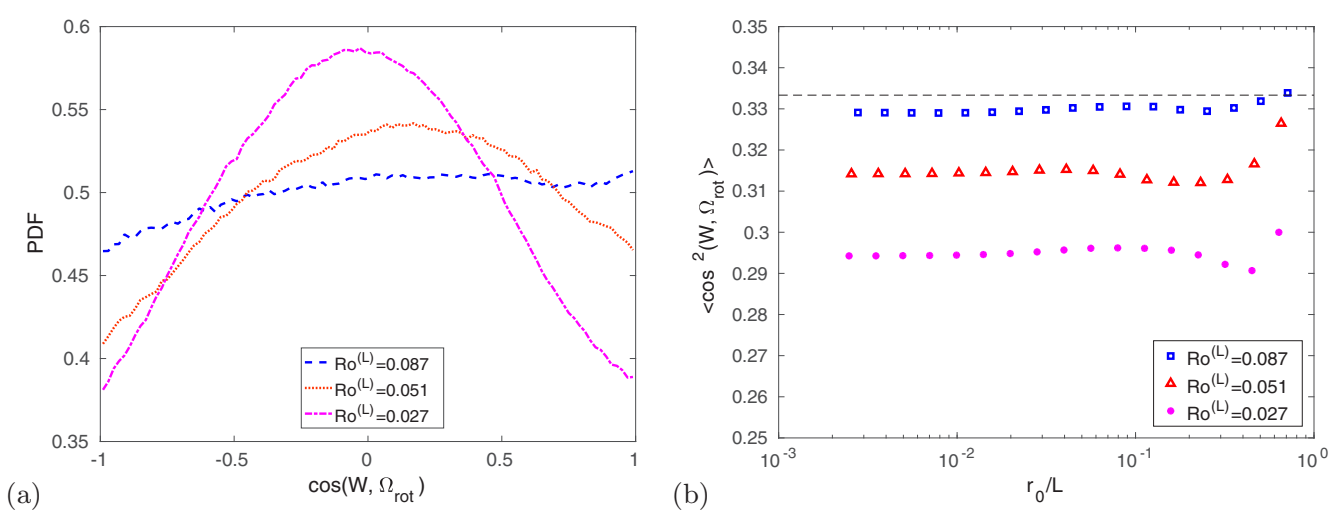

FIG. 2. (a) PDF of the cosine of the angle between vortex stretching vector $\mathbf{W}\left(r_{0}\right)$ and rotation vector $\boldsymbol{\Omega}_{\text {rot }}$ for $r_{0} \lesssim \eta$ and different values of the Rossby number. (b) Scale dependence of $\left\langle\cos ^{2}\left[\mathbf{W}\left(r_{0}\right), \boldsymbol{\Omega}_{\text {rot }}\right]\right\rangle$ for different $\mathrm{Ro}^{(L)}$. The horizontal line indicates the value $1 / 3$.

to be reduced by rotation, a suggestion that will be confirmed later on. As rotation is decreased, the preferential horizontality of $\mathbf{W}$ is diminished: For $\operatorname{Ro}^{(L)}=0.087$ the PDF of $\cos \left[\mathbf{W}\left(r_{0}\right), \boldsymbol{\Omega}_{\text {rot }}\right]$ is almost flat, which would correspond to a random orientation of the two vectors. Nevertheless, for $\mathrm{Ro}^{(L)}=0.051$ and 0.087 the vectors seem to point preferentially towards the same direction $\left[\left\langle\cos \left(\mathbf{W}, \boldsymbol{\Omega}_{\text {rot }}\right)\right\rangle\right.$ is slightly positive $]$.

Multiscale information is provided by Fig. 2(b), which displays the scale dependence of the second-order moment of $\cos \left[\mathbf{W}\left(r_{0}\right), \boldsymbol{\Omega}_{\text {rot }}\right]$ for the three rotating runs. This quantity allows us to distinguish cases where the two vectors are randomly oriented with respect to each other $\left[\left\langle\cos ^{2}\left(\mathbf{W}, \boldsymbol{\Omega}_{\text {rot }}\right)\right\rangle=1 / 3\right]$ from those where they have a propensity to be collinear $\left[\left\langle\cos ^{2}\left(\mathbf{W}, \boldsymbol{\Omega}_{\text {rot }}\right)\right\rangle>\right.$ $1 / 3]$ or normal $\left[\left\langle\cos ^{2}\left(\mathbf{W}, \boldsymbol{\Omega}_{\text {rot }}\right)\right\rangle<1 / 3\right]$. For the three runs, Fig. 2(b) shows that $\left\langle\cos ^{2}\left(\mathbf{W}, \boldsymbol{\Omega}_{\text {rot }}\right)\right\rangle \rightarrow$ $1 / 3$ as $r_{0} \rightarrow L$, as expected since both vectors become independent of each other above this scale. For any scale $r_{0}<L$ and any Rossby number, they are (in a statistical sense) perpendicular to each other, a tendency getting stronger at decreasing $\mathrm{Ro}^{(L)}$, that is, as rotation effects become more important, and also visible at small scale in Fig. 2(a). Moreover, $\left\langle\cos ^{2}\left(\mathbf{W}, \boldsymbol{\Omega}_{\text {rot }}\right)\right\rangle$ reaches a minimum, that is, the preferential normality between vortex stretching vector and rotation axis is maximal, at a scale approximately equal to $L / 3$ which seems to depend weakly on the Rossby number and is much larger than $\ell_{\mathrm{HZ}}$ and $\ell_{d}$ (see Table I). It is worthwhile recalling here that the cyclone-anticyclone antisymmetry and related collinearity between $\boldsymbol{\Omega}_{\mathrm{rot}}$ and $\boldsymbol{\Omega}\left(r_{0}\right)$ have been found to be maximal at a similar scale $[19,37,43]$. These results will be discussed in Sec. IV.

\section{Orientation between rate of strain and rotation vector}

We now investigate the relative orientation properties between rotation and the rate-of-strain tensor eigenframe by examining the statistics of $\cos \left[\hat{\mathbf{S}}_{i}\left(r_{0}\right), \boldsymbol{\Omega}_{\mathrm{rot}}\right]$ for $i=1,2,3$. As expected, since the direction of an eigenvector is undefined, the PDF of this quantity is symmetric and its average is zero for any $\mathrm{Ro}^{(L)}$ and $r_{0}$. The variance of $\cos \left(\hat{\mathbf{S}}_{i}, \boldsymbol{\Omega}_{\text {rot }}\right)$ is plotted as a function of scale and for all rotating runs in Fig. 3. Here $\hat{\mathbf{S}}_{2}$ is found to be preferentially collinear to the rotation vector (i.e., vertical) and $\hat{\mathbf{S}}_{3}$ to be preferentially perpendicular to it (horizontal). These tendencies are more and more pronounced as the Rossby number is decreased, that is, when rotation gets more intense. The orientation of the first eigenvector $\hat{\mathbf{S}}_{1}$ is less universal: For the two largest Rossby numbers, it tends to be vertical at large scales and horizontal at small ones, whereas for $\mathrm{Ro}^{(L)}=0.027$ it is horizontal (in a statistical sense) for any $r_{0}$. However, these trends are significantly less pronounced than those evidenced for $\hat{\mathbf{S}}_{2}$ and $\hat{\mathbf{S}}_{3}$. 

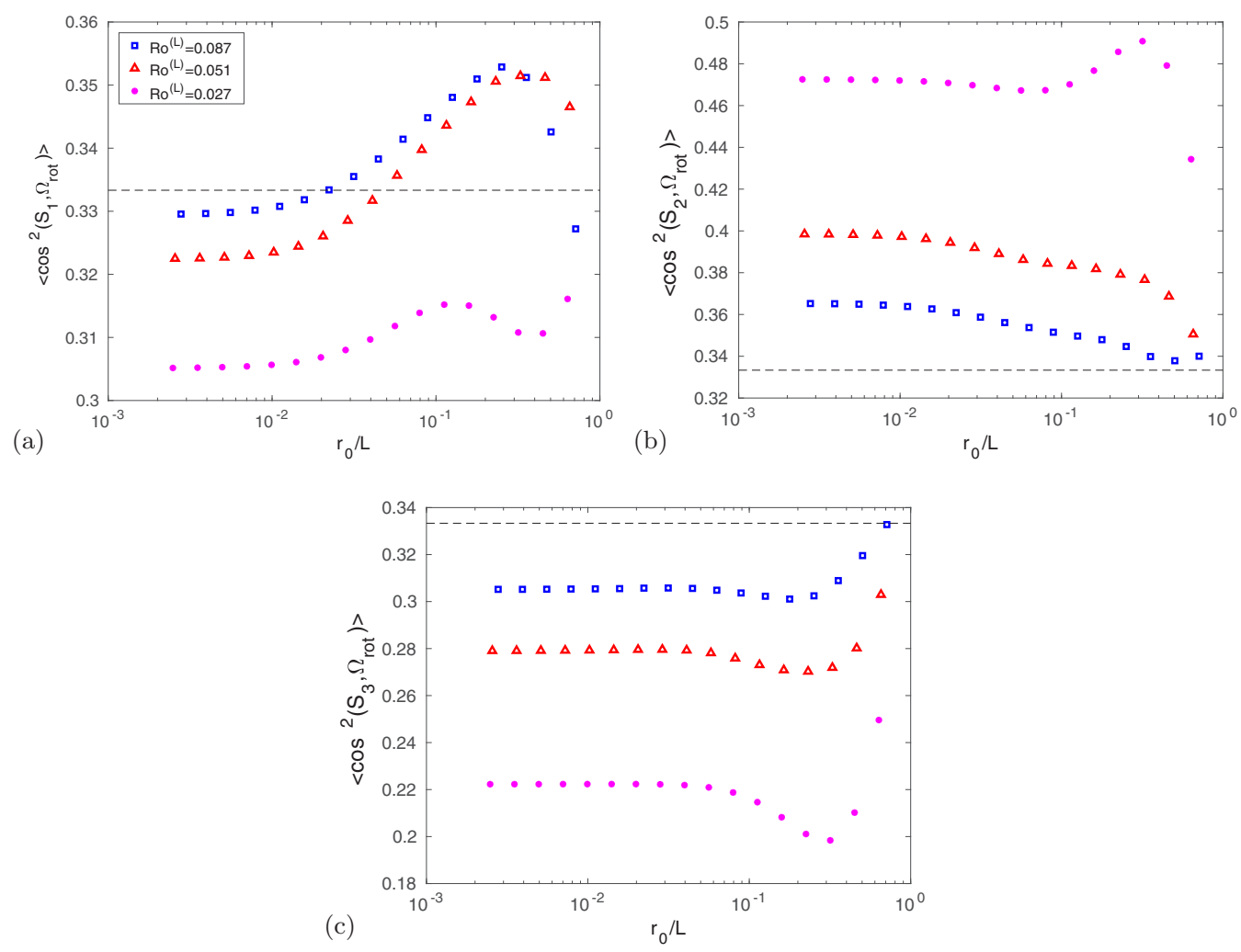

FIG. 3. Scale dependence of $\left\langle\cos ^{2}\left[\hat{\mathbf{S}}_{i}\left(r_{0}\right), \boldsymbol{\Omega}_{\mathrm{rot}}\right]\right\rangle$ for (a) $i=1$ (eigenvector associated with the largest rate of strain), (b) $i=2$ (intermediate eigenvector), and (c) $i=3$ (eigenvector associated with the smallest rate of strain). Blue squares denote $\operatorname{Ro}^{(L)}=0.087$, red triangles $\operatorname{Ro}^{(L)}=0.051$, and purple circles $\operatorname{Ro}^{(L)}=0.027$. Horizontal lines indicate the value $1 / 3$.

At the smallest scales, these results display qualitative agreement with those obtained experimentally in [38]. As mentioned therein, the growing tendency for $\hat{\mathbf{S}}_{1}$ (largest stretching direction) and $\hat{\mathbf{S}}_{3}$ (compression direction) to lie in the horizontal plane as rotation effects increase is related to the two-dimensionalization of the flow induced by rotation.

When $r_{0} \rightarrow L,\left\langle\cos ^{2}\left[\hat{\mathbf{S}}_{i}\left(r_{0}\right), \boldsymbol{\Omega}_{\mathrm{rot}}\right]\right\rangle \rightarrow 1 / 3(i=1,2,3)$, that is, all the strain eigenvectors tend to be randomly oriented, as expected. Finally, the maximal deviation from a random orientation of the strain eigenframe occurs, for any eigenvector of $\mathbf{S}$ and any Rossby number, at a scale approximately equal to $L / 3$, similarly to what was observed for the vortex stretching vector (Sec. III A 1 ) and vorticity [37]. Such a result will be interpreted later on.

\section{B. Statistical properties of the strain tensor}

The statistical properties of the strain tensor are now addressed. Sections III B 1 and III B 2 will be respectively devoted to the analysis of the normalized eigenvalues $S_{1}, S_{2}$, and $S_{3}$ and of the orientation between the rate-of-strain eigenframe and vorticity.

\section{Eigenvalues}

The PDFs of the normalized eigenvalues $\tilde{S}_{i} \equiv S_{i} /\left[\operatorname{Tr}\left(\mathbf{S}^{2}\right)\right]^{1 / 2}(i=1,2,3)$ are plotted in Fig. 4(a) for the smallest value of $r_{0}(\lesssim \eta)$ and (in the inset) the largest one $\left(r_{0} \lesssim L\right)$. The shapes of the distributions vary continuously with $r_{0}$ for all the flows. In isotropic turbulence (solid lines), the 
(a)
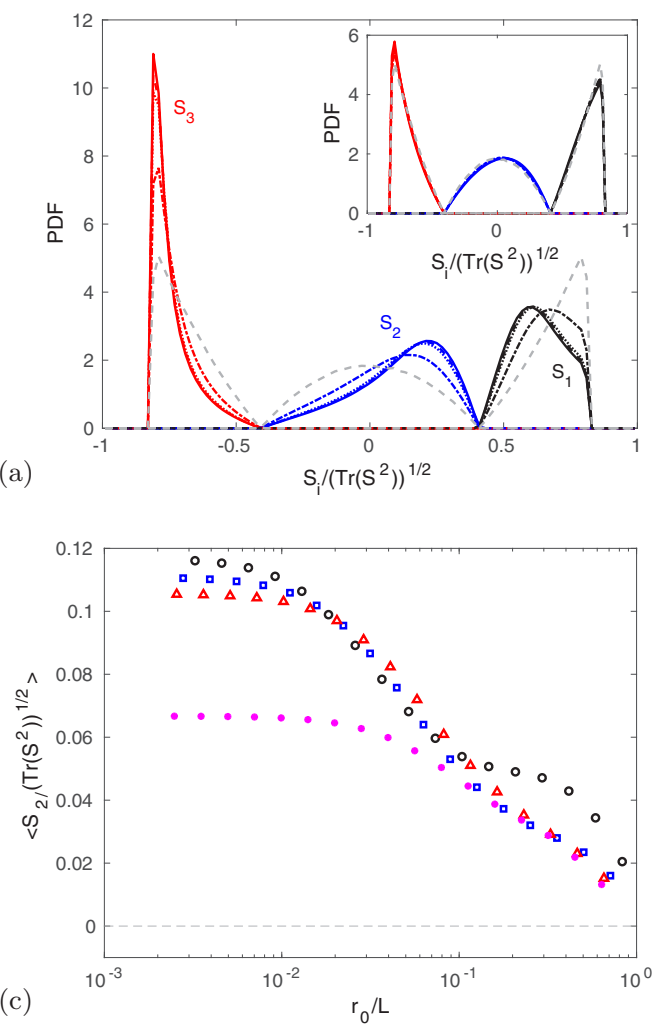
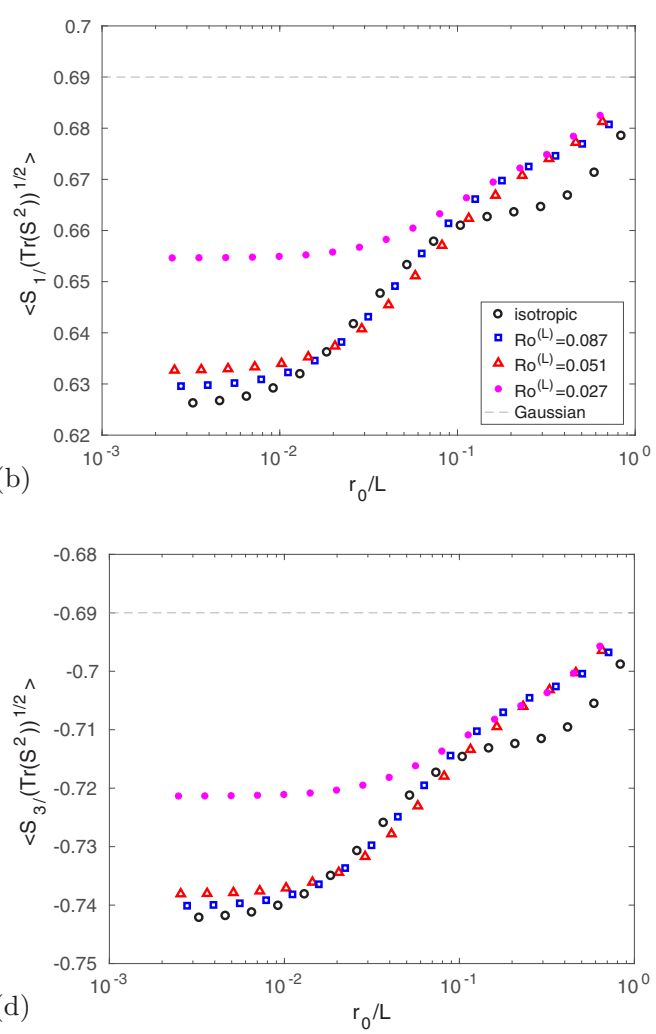

FIG. 4. (a) PDF of the normalized eigenvalues of strain $\tilde{S}_{i}=S_{i} /\left[\operatorname{Tr}\left(\mathbf{S}^{2}\right)\right]^{1 / 2}$ for different values of the Rossby number: isotropic (solid lines), $\mathrm{Ro}^{(L)}=0.087$ (dashed lines), $\mathrm{Ro}^{(L)}=0.051$ (dotted lines), and $\mathrm{Ro}^{(L)}=0.027$ (dash-dotted lines), with $r_{0} \lesssim \eta$ in the main panel (in the inset $r_{0} \lesssim L$ ). Also shown is the scale dependence of the mean normalized eigenvalues of strain with (b) $i=1$ (largest eigenvalue), (c) $i=2$ (intermediate eigenvalue), and (d) $i=3$ (smallest eigenvalue) for different values of the Rossby number: isotropic (open black circles), $\operatorname{Ro}^{(L)}=0.087$ (blue squares), $\operatorname{Ro}^{(L)}=0.051$ (red triangles), $\operatorname{Ro}^{(L)}=0.027$ (closed purple circles). In the four panels the light gray lines indicate the values for a Gaussian ensemble of matrices $\mathbf{M}$.

PDF of $\tilde{S_{2}}=S_{2} /\left[\operatorname{Tr}\left(\mathbf{S}^{2}\right)\right]^{1 / 2}$ is, as expected, strongly biased towards positive values for $r_{0} \lesssim \eta[7-9]$. This bias reduces as the scale is increased: For $r_{0} \lesssim L$ the distribution is almost symmetric (it would be exactly symmetric for a Gaussian ensemble of matrices; see dashed gray lines). The shapes displayed by the PDF of $\tilde{S}_{2}$ are similar to those obtained numerically in [13] (see also [44] in the dissipative range). For the smallest $r_{0}$ considered, the PDF of $\tilde{S_{3}}$ is much more peaked than that of $\tilde{S_{1}}$.

As rotation effects increase (i.e., at decreasing $\mathrm{Ro}^{(L)}$ ), the three distributions get closer to the Gaussian ones at all scales. In particular, the PDF of $\tilde{S_{2}}$ is less and less skewed, that is, $\left\langle S_{2}\right\rangle \rightarrow 0$, a result that will be confirmed later on. Such a Gaussianization of turbulence under the effect of rotation is well known in other contexts $[45,46]$. The effect of rotation on the distributions of the non-normalized eigenvalues $S_{i}$ (not shown here) is the suppression of their large tails for $S_{1}$ and $S_{3}$ and skewness for $S_{2}$. In the dissipative range (smallest $r_{0}$ ), these behaviors were also reported in [38].

More quantitative information is provided by the averaged normalized eigenvalues $\left\langle\tilde{S}_{i}\right\rangle$ plotted as a function of $r_{0}$ in Figs. 4(b)-4(d). We first consider the flow without rotation. At the smallest scale, $\left\langle\tilde{S}_{2}\right\rangle>0$, as widely known in the dissipative range [7-9]. As already mentioned, a consequence of this result is the self-amplification of strain. In the same regime, $\left\langle\tilde{S}_{1}\right\rangle$ and $\left\langle\tilde{S}_{3}\right\rangle$ are both smaller than the values they would have for a Gaussian ensemble of matrices. At increasing scale the three averages tend to the Gaussian values predicted for $r_{0} \gtrsim L$. The slopes of the three curves are 

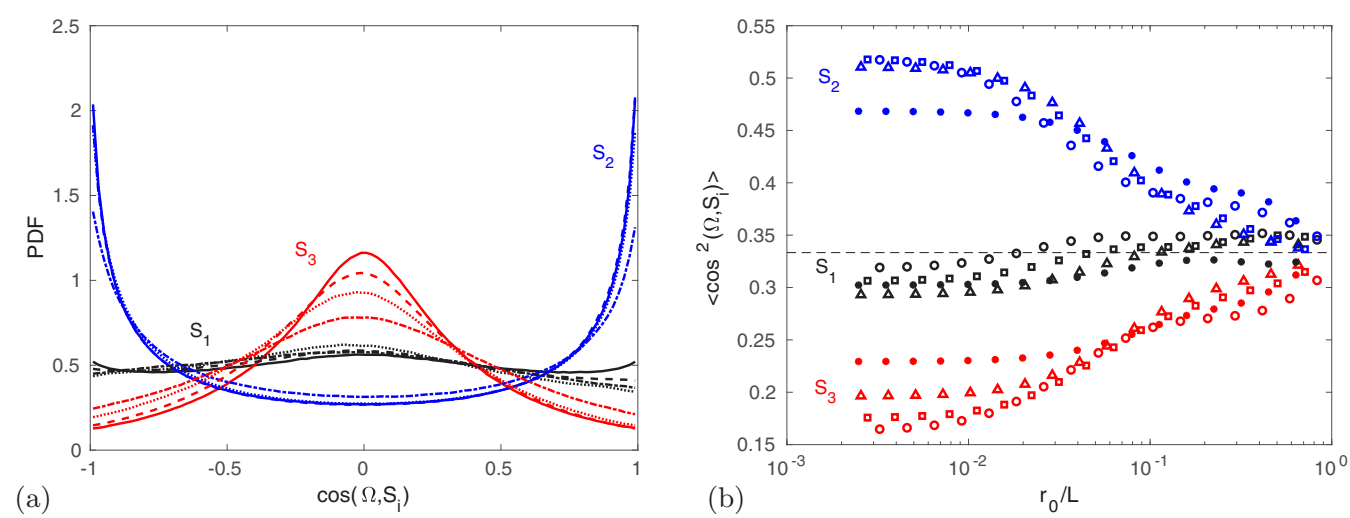

FIG. 5. (a) PDF of the cosine between vorticity $\boldsymbol{\Omega}\left(r_{0}\right)$ and the eigenvectors of the rate of strain $\hat{\mathbf{S}}_{i}\left(r_{0}\right)$ for $r_{0} \lesssim \eta$ and different values of the Rossby number: isotropic (solid lines), $\mathrm{Ro}^{(L)}=0.087$ (dashed lines), $\operatorname{Ro}^{(L)}=0.051$ (dotted lines), and $\mathrm{Ro}^{(L)}=0.027$ (dash-dotted lines). (b) Scale dependence of $\left\langle\cos ^{2}\left(\boldsymbol{\Omega}, \hat{\mathbf{S}}_{i}\right)\right\rangle$ for different Rossby numbers: isotropic (open circles), $\mathrm{Ro}^{(L)}=0.087$ (squares), $\mathrm{Ro}^{(L)}=0.051$ (triangles), and $\mathrm{Ro}^{(L)}=0.027$ (closed circles). The horizontal line indicates the value $1 / 3$.

discontinuous for $r_{0} \sim L / 10$. At higher Reynolds number, this discontinuity would be replaced by a plateau indicating the inertial range of scales, as shown for $\left\langle\tilde{S}_{2}\right\rangle$ in [13]. More generally, the results displayed in Fig. 4 for $\tilde{S_{2}}$ in the isotropic flow are in agreement with those obtained numerically in [13] (the statistics of $\tilde{S_{1}}$ and $\tilde{S_{3}}$ were not investigated in this work).

In the presence of rotation, the values of the averaged eigenvalues are closer to the Gaussian expectations at any scale, a feature getting more and more prominent as rotation is increased. Such a result is in agreement with Fig. 4(a) and is related to the above-mentioned Gaussianization of turbulence under the effect of rotation. This statistical orientation is also associated with the twodimensionalization of turbulence by rotation: In strictly 2D turbulence, $S_{2}$ would be strictly zero (that is, strain would not self-amplify) and the two other eigenvalues $S_{1}$ and $S_{3}$ would be opposite to each other. At the smallest scale, our results are similar to those of [38]. Incidentally, it can be noticed that for rotating flows the variations of $\left\langle\tilde{S}_{i}\right\rangle(i=1,2,3)$ are continuous, the inertial range of scales being narrower than in the isotropic flow, which will be confirmed in Sec. III C 2. In particular, the scales $\ell_{\mathrm{HZ}}$ and $\ell_{d}$ do not seem to play any role for these statistics.

\section{Orientation between vorticity and strain}

We now turn to the analysis of orientation statistics between the rate-of-strain eigenbasis $\hat{\mathbf{S}}_{i}\left(r_{0}\right)$ and vorticity $\boldsymbol{\Omega}\left(r_{0}\right)$. Figure 5(a) shows the PDFs of $\cos \left(\boldsymbol{\Omega}, \hat{\mathbf{S}}_{i}\right)$ at the smallest scale and for all the flows considered. These distributions are obviously symmetric. The isotropic flow is first discussed here. As widely known in HIT in the dissipative range of scales [5-9], the eigenvector associated with the intermediate eigenvalue, $\hat{\mathbf{S}}_{2}$, is preferentially collinear with vorticity, while the one associated with the stretching direction, $\hat{\mathbf{S}}_{3}$, tends to be normal to it and $\hat{\mathbf{S}}_{1}$ is slightly perpendicular to $\boldsymbol{\Omega}$. At increasing scale, these orientation statistics weaken, as illustrated in Fig. 5(b), and the distributions (not shown here) become more and more flat, as expected for a Gaussian ensemble of matrices and also observed in [13], with which very good qualitative agreement is obtained.

In the presence of rotation, this statistical alignment is also reduced, at any scale, as visible in Figs. 5(a) and 5(b). This is once more a signature of the Gaussianization of turbulence by rotation. On the other hand, this result may seem at first sight contradictory with the statistical orientation of the strain-rate eigenframe and of vorticity with the rotation axis. It was indeed shown in [37] that vorticity $\boldsymbol{\Omega}$ was preferentially vertical and in Sec. III A 2 of the present paper that $\hat{\mathbf{S}}_{2}$ (respectively $\hat{\mathbf{S}}_{3}$ ) tends to be vertical (respectively horizontal). A simple reasoning would lead to 

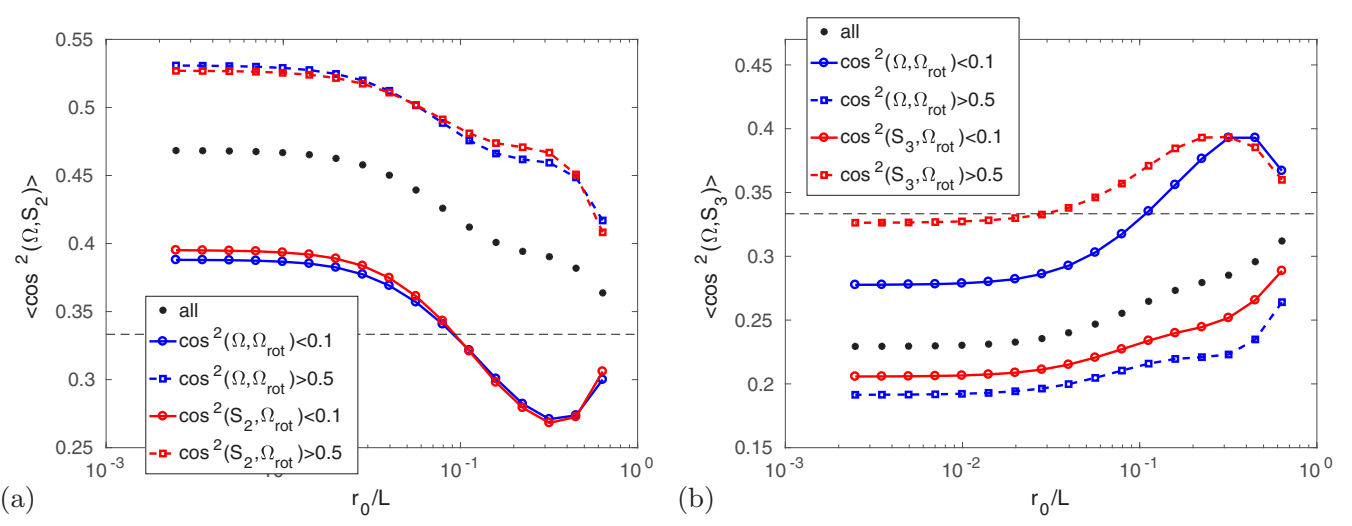

FIG. 6. Scale dependence of (a) $\left\langle\cos ^{2}\left[\boldsymbol{\Omega}\left(r_{0}\right), \hat{\mathbf{S}}_{2}\left(r_{0}\right)\right]\right\rangle$ and (b) $\left\langle\cos ^{2}\left[\boldsymbol{\Omega}\left(r_{0}\right), \hat{\mathbf{S}}_{3}\left(r_{0}\right)\right]\right\rangle$ conditioned on the magnitude of $\cos ^{2}\left[\boldsymbol{\Omega}\left(r_{0}\right), \boldsymbol{\Omega}_{\mathrm{rot}}\right]$ and of $\cos ^{2}\left[\hat{\mathbf{S}}_{i}\left(r_{0}\right), \boldsymbol{\Omega}_{\mathrm{rot}}\right]$, for $\mathbf{R o}^{(L)}=0.027$. Horizontal lines indicate the value $1 / 3$.

the conclusion that rotation reinforces collinearity (respectively perpendicularity) of $\boldsymbol{\Omega}$ with $\hat{\mathbf{S}}_{2}$ (respectively $\hat{\mathbf{S}}_{3}$ ). However, it is worth recalling here that these properties hold in a statistical sense only. To clarify the apparent contradiction, we plot in Fig. 6 , for $i=2,3, \operatorname{Ro}^{(L)}=0.027$, and all the scales $r_{0}$ considered, the variance of $\cos \left(\boldsymbol{\Omega}, \hat{\mathbf{S}}_{i}\right)$ conditioned on the orientation of the eigenvector $\hat{\mathbf{S}}_{i}$ and of vorticity with respect to the rotation axis (the results for the two other rotating flows are qualitatively similar, although the trends are then naturally less pronounced). Figure 6(a) shows that $\hat{\mathbf{S}}_{2}$ and $\boldsymbol{\Omega}$ are preferentially collinear when one or the other vector is more vertical. Similarly, it is shown in Fig. 6(b) that the perpendicularity of $\hat{\mathbf{S}}_{3}$ and $\boldsymbol{\Omega}$ is favored when the latter (former) is statistically more vertical (horizontal). Remarkably, these features hold at any scale. They are however the most stringent for $r_{0} \approx L / 3$, at which the statistical orientation of dynamical quantities with the rotating frame is the strongest. These observations allow us to suggest the following interpretation: While turbulence is two-dimensionalized in some regions of the flow (which are certainly the columnar structures reminiscent of Taylor columns and associated with the slow modes mentioned in the Introduction), statistical alignment between vorticity and the strain-rate eigenbasis is suppressed in the rest of the fluid, probably under the effect of inertial waves. A common way to test this interpretation would consist in performing the same analysis on a decomposed velocity field in which the fast manifold (three dimensional, associated with waves) is separated from the slow manifold (two dimensional, corresponding to pure vortical motion and large-scale columnar structures). Such a decomposition was recently used in [32]. Overall, it seems that the regions in which statistical alignment between vorticity and the strain-rate eigenbasis is suppressed dominate the statistics of orientation between $\boldsymbol{\Omega}$ and the strain eigenframe in the three rotating flows calculated here.

On a side note, the results displayed in Fig. 5 at the smallest scale differ from the experimental data presented in [38], which rather show (in the dissipative range) an amplification of collinearity (respectively perpendicularity) of $\hat{\mathbf{S}}_{2}$ (respectively $\hat{\mathbf{S}}_{3}$ ) with vorticity under the effect of rotation. Our results are however similar to those obtained by direct numerical simulation, in the dissipative range, in [47]. Following the interpretation proposed above, this disagreement might be due to the fact that columnar structures cover a larger part of the flow, or that the influence of inertial waves is weaker, in the experiments of [38] than in our simulations and those of [47].

\section{Statistics related to the mechanisms of enstrophy production and strain production}

\section{Orientation between vorticity and vortex stretching vector}

We now investigate the statistical orientation between the vorticity $\boldsymbol{\Omega}\left(r_{0}\right)$ and vortex stretching vector $\mathbf{W}\left(r_{0}\right)$. The PDFs of the cosine of the angle between both vectors is displayed in Figs. 7(a) and 

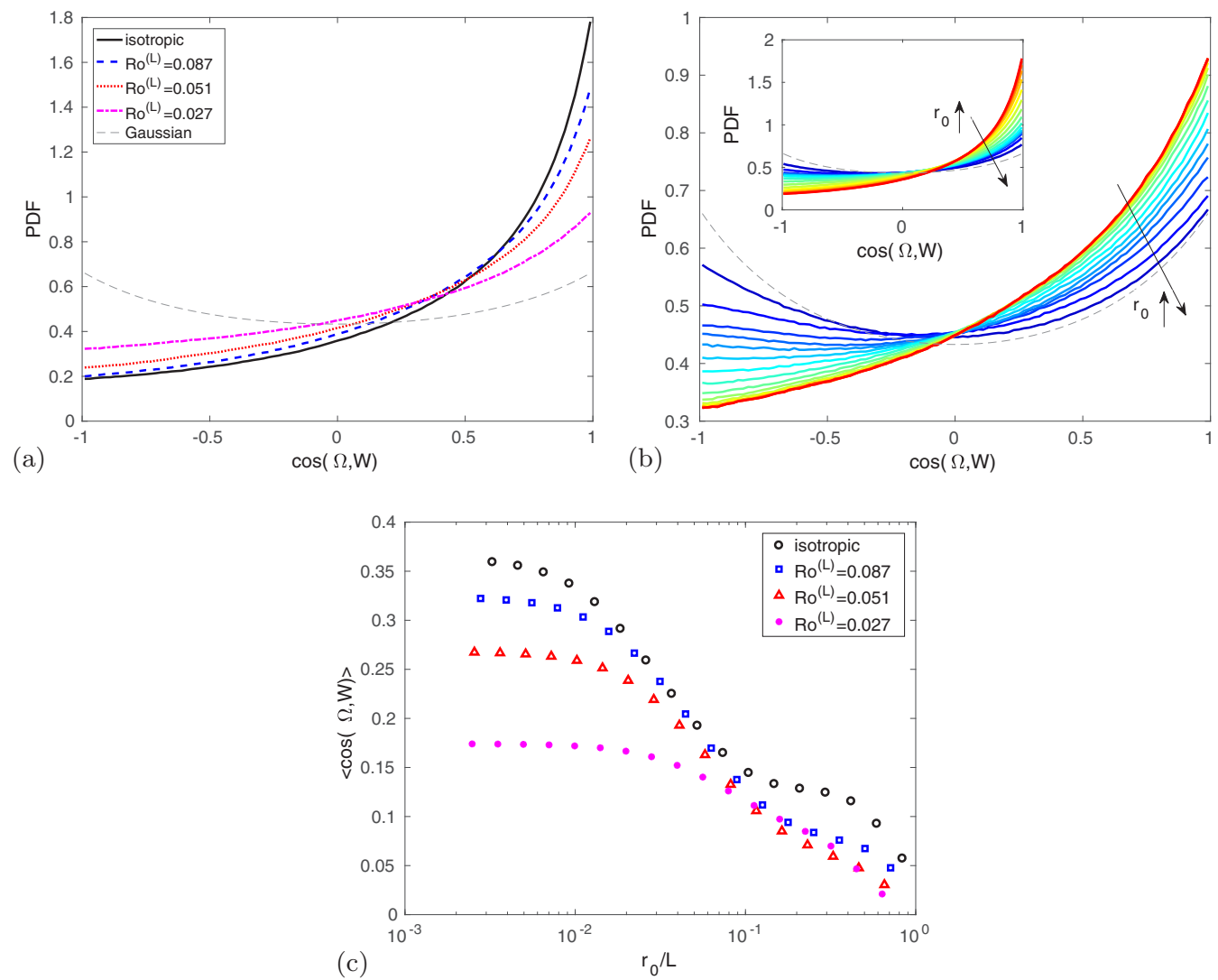

FIG. 7. PDF of the cosine between vorticity $\boldsymbol{\Omega}\left(r_{0}\right)$ and vortex stretching vector $\mathbf{W}\left(r_{0}\right)$ for (a) $r_{0} \lesssim \eta$ and different values of the Rossby number and (b) different values of $r_{0}$ (increasing values from red to blue) and $\mathrm{Ro}^{(L)}=0.027$ in the main panel (or without rotation in the inset). The light gray lines are the distributions expected for a Gaussian ensemble of matrices M. (c) Scale dependence of $\left\langle\cos \left[\boldsymbol{\Omega}\left(r_{0}\right), \mathbf{W}\left(r_{0}\right)\right]\right\rangle$ for different values of $\mathrm{Ro}^{(L)}$.

7(b). The distributions are plotted for the smallest scale and different values of Ro ${ }^{(L)}$ in Fig. 7(a) and for all scales and two flows in Fig. 7(b) $\left(\mathrm{Ro}^{(L)}=0.027\right.$ in the main panel, no rotation in the inset).

When rotation is turned off, the distributions exhibit the well known features of 3D HIT: At small scale (in the dissipative range) the probability of positive events (associated with stretching) is much higher than that of negative (compression) events. Overall, vortex stretching therefore dominates [5-8]. From a geometrical point of view, $\boldsymbol{\Omega}$ and $\mathbf{W}$ preferentially point towards the same direction. At increasing scale, this asymmetry is diminished until disappearing completely at the integral scale [see the dashed gray line in the inset of Fig. 7(b)], above which the velocities at the tetrahedron vertices are uncorrelated.

Increasing the rotation rate reduces statistical alignment between $\boldsymbol{\Omega}$ and $\mathbf{W}$ [see Fig. 7(a) and compare the main panel with the inset of Fig. 7(b)] and thereby the net enstrophy production, a result that will be confirmed later on. This tendency is a signature of the associated Gaussianization and two-dimensionalization of turbulence by rotation [23] and was reported, in the dissipative range, experimentally [38,48] and numerically [47]. It is also consistent (even though caution must be taken about the statistical nature of these properties; see, e.g., Sec. III B 2) with the fact that when rotation is activated, $\boldsymbol{\Omega}$ tends to be vertical [37] whereas $\mathbf{W}$ is preferentially horizontal (Sec. III A 1).

All these trends are also illustrated more quantitatively in Fig. 7(c), in which the scale dependence of $\langle\cos (\boldsymbol{\Omega}, \mathbf{W})\rangle$ is plotted for the four simulations performed. Once more, the formation of an inertial 

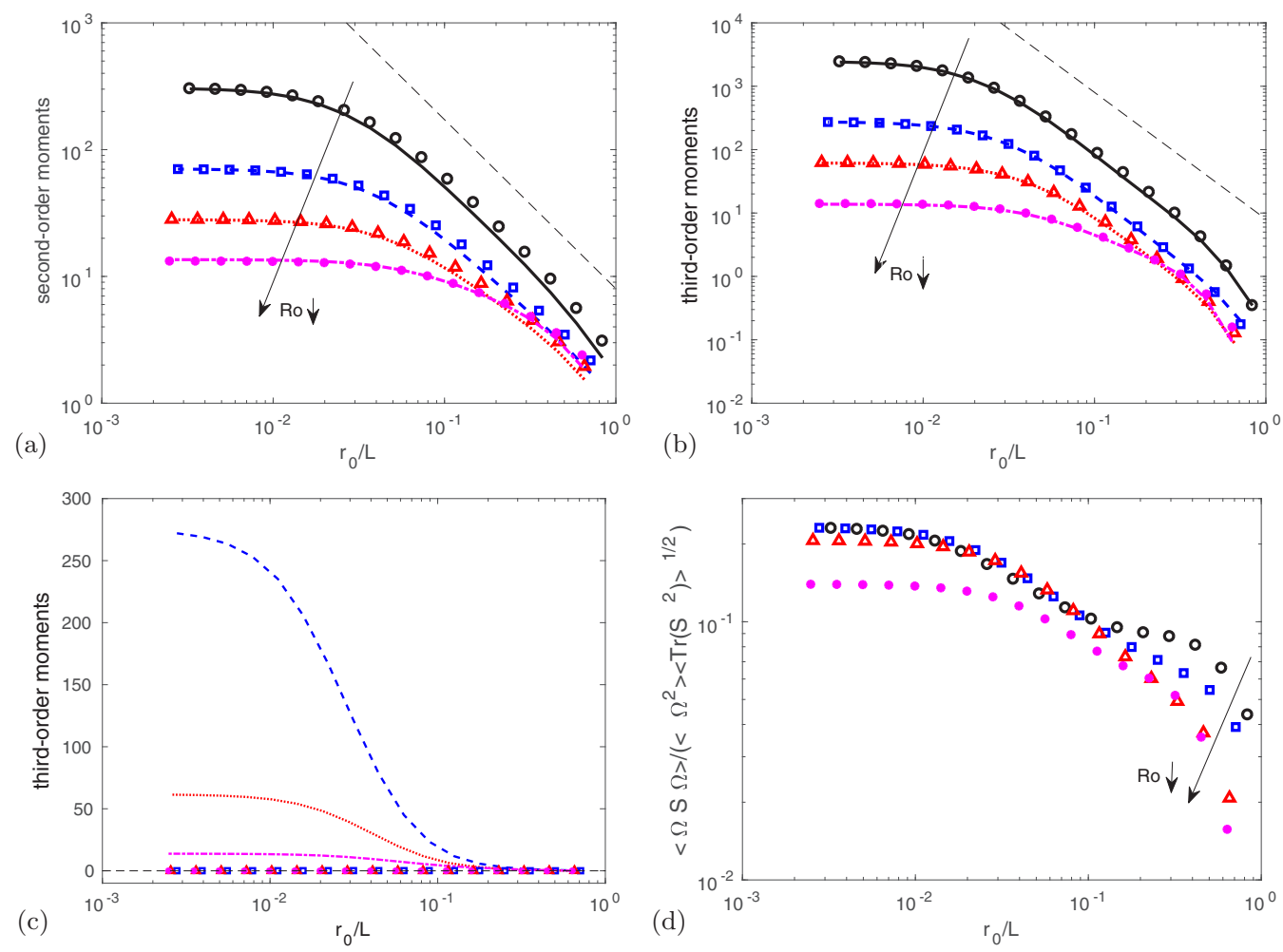

FIG. 8. Scale dependence of the low-order moments of the perceived velocity gradient tensor $\mathbf{M}$ for different values of $\operatorname{Ro}^{(L)}$ : (a) second-order moments $\left\langle\boldsymbol{\Omega}^{2}\right\rangle / 2$ (lines) and $\left\langle\operatorname{Tr}\left(\mathbf{S}^{2}\right)\right\rangle$ (symbols), (b) third-order moments $\langle\boldsymbol{\Omega} \mathbf{S} \boldsymbol{\Omega}\rangle$ (lines) and $-(4 / 3)\left\langle\operatorname{Tr}\left(\mathbf{S}^{3}\right)\right\rangle$ (symbols), (c) third-order moments $\langle\boldsymbol{\Omega} \mathbf{S} \boldsymbol{\Omega}\rangle$ (lines) and $2\left\langle\boldsymbol{\Omega}_{\text {rot }} \mathbf{S} \boldsymbol{\Omega}\right\rangle$ (symbols), and (d) normalized enstrophy production $\langle\boldsymbol{\Omega} \mathbf{S} \boldsymbol{\Omega}\rangle /\left[\left\langle\boldsymbol{\Omega}^{2}\right\rangle\left\langle\operatorname{Tr}\left(\mathbf{S}^{2}\right)\right\rangle^{1 / 2}\right]$. In (a) and (b) the straight lines indicate the slopes $-4 / 3$ and -2 , respectively. In all panels are the following values of the Rossby number: isotropic (open black circles), $\mathrm{Ro}^{(L)}=0.087$ (blue squares), $\mathrm{Ro}^{(L)}=0.051$ (red triangles), and $\mathrm{Ro}^{(L)}=0.027$ (closed purple circles).

range of scales can be distinguished for the nonrotating flow only and no effect of the HopfingerZeman and $\ell_{d}$ scales is visible in the rotating flows.

\section{Scaling laws of the low-order moments of the perceived velocity gradient tensor}

The present section is devoted to the measurement of low-order moments of the tensors $\mathbf{S}$ and $\boldsymbol{\Omega}$ and of their physically relevant products. Figure 8(a) first displays the scaling laws of second-order moments, half the enstrophy $\left\langle\boldsymbol{\Omega}^{2}\right\rangle / 2$ and rate-of-strain variance $\left\langle\operatorname{Tr}\left(\mathbf{S}^{2}\right)\right\rangle$, for the four flows simulated. At small scale both quantities are equal, as expected in the dissipative range in homogeneous turbulence. This equality is found to be approximately satisfied at any scale. In the absence of rotation, a range of scales in which the Kolmogorov prediction $\left\langle\mathbf{M}^{2}\right\rangle \sim r_{0}^{-2 / 3}$ is well satisfied can be distinguished. At increasing rotation this inertial range progressively disappears and the magnitudes of both second-order moments decrease.

We now focus on the enstrophy and strain production terms. In the absence of external forces, the Lagrangian dynamics of the usual enstrophy and rate-of-strain variance are written, respectively, as

$$
\frac{1}{2} \frac{D\left(\omega^{2}\right)}{D t}=\omega_{i} s_{i j} \omega_{j}+v \omega_{i} \partial_{j j} \omega_{i}
$$




$$
\frac{1}{2} \frac{D \operatorname{Tr}\left(\boldsymbol{s}^{2}\right)}{D t}=-\operatorname{Tr}\left(\boldsymbol{s}^{3}\right)-\frac{1}{4} \boldsymbol{\omega} \boldsymbol{\omega} \boldsymbol{\omega}-\frac{1}{\rho} s_{i j} \partial_{i j} p+\nu s_{i j} \partial_{k k} s_{i j},
$$

where $D / D t$ denotes the Lagrangian time derivative. The production terms of enstrophy and strain are therefore $\langle\omega \mathbf{S} \boldsymbol{\omega}\rangle$ and $-\left\langle\operatorname{Tr}\left(\mathbf{s}^{3}\right)\right\rangle-\langle\omega \mathbf{S} \boldsymbol{\omega}\rangle / 4$, respectively. In a rotating frame, these terms become $\left\langle\left(\boldsymbol{\omega}+2 \boldsymbol{\Omega}_{\text {rot }}\right) \mathbf{s} \boldsymbol{\omega}\right\rangle$ and $-\left\langle\operatorname{Tr}\left(\mathbf{s}^{3}\right)\right\rangle-\left\langle\left(\boldsymbol{\omega}+4 \boldsymbol{\Omega}_{\text {rot }}\right) \mathbf{s} \boldsymbol{\omega}\right\rangle / 4$ [35,38]. The transport equations of the perceived enstrophy and strain variance are expected to be more complicated than Eqs. (4) and (5) and to include in particular subgrid terms. These equations will not be written here. We will rather focus on the statistics of quantities depending explicitly on the perceived velocity gradient tensor $\mathbf{M}\left[\mathbf{\Omega S} \boldsymbol{\Omega},-\operatorname{Tr}\left(\mathbf{S}^{3}\right), \boldsymbol{\Omega}_{\mathrm{rot}} \mathbf{S} \boldsymbol{\Omega}\right.$, and their sums], abusively referring to them as perceived enstrophy or strain variance. Such an approach was already used in [35].

The scaling laws of "isotropic" third-order moments, $\langle\boldsymbol{\Omega S} \boldsymbol{\Omega}\rangle$ ("enstrophy production") and $-(4 / 3)\left\langle\operatorname{Tr}\left(\mathbf{S}^{3}\right)\right\rangle$ ("strain production"), are plotted in Fig. 8(b). These terms are approximately equal to each other at all scales (in homogeneous flows, the equality is expected to be strict in the dissipative range). Similarly to second-order moments, the amplitudes of $\langle\boldsymbol{\Omega} \mathbf{S} \boldsymbol{\Omega}\rangle$ and $-(4 / 3)\left\langle\operatorname{Tr}\left(\mathbf{S}^{3}\right)\right\rangle$ decrease with decreasing Rossby number, i.e., as rotation effects get stronger (the same effect was reported in [38] in the dissipative range). In the statistically isotropic flow, an inertial range, in which the Kolmogorov scaling $\sim r_{0}^{-2}$ holds, is clearly visible.

As previously mentioned, the total enstrophy production in rotating flows is $\left\langle\left(\boldsymbol{\Omega}+2 \boldsymbol{\Omega}_{\mathrm{rot}}\right) \mathbf{S} \boldsymbol{\Omega}\right\rangle$. The two terms involved in this expression are plotted as a function of $r_{0}$ in Fig. 8(c) for the three anisotropic flows simulated. In all cases, $\left|\left\langle\boldsymbol{\Omega}_{\mathrm{rot}} \mathbf{S} \boldsymbol{\Omega}\right\rangle\right| \ll|\langle\boldsymbol{\Omega} \mathbf{S} \boldsymbol{\Omega}\rangle|$. As a consequence, the latter ("isotropic") production term reasonably approximates the full contribution. A similar conclusion can be drawn for the rate of strain, i.e., $-\left\langle\operatorname{Tr}\left(\mathbf{S}^{3}\right)\right\rangle-\langle\boldsymbol{\Omega} \mathbf{S} \boldsymbol{\Omega}\rangle / 4$ is a very satisfactory estimate of the total strain production. In particular, according to Fig. 8(b), the total enstrophy and strain production drastically decrease, at any scale, as rotation is increased.

To go further, we show in Fig. 8(d) the scale dependence of the enstrophy production rate, defined as $\langle\boldsymbol{\Omega} \boldsymbol{S} \boldsymbol{\Omega}\rangle /\left[\left\langle\boldsymbol{\Omega}^{2}\right\rangle\left\langle\operatorname{Tr}\left(\mathbf{S}^{2}\right)\right\rangle^{1 / 2}\right] .^{2}$ A small plateau associated with the inertial range seems to be present for the isotropic flow. For any $r_{0}$, the enstrophy production rate is strongly reduced with increasing rotation. This feature reflects once more the Gaussianization and two-dimensionalization of turbulence under the effect of rotation and is related to the dealignment between $\boldsymbol{\Omega}$ and $\mathbf{W}$ evidenced in Sec. III C 1. Using the fact that $\left\langle-\operatorname{Tr}\left(\mathbf{S}^{3}\right)\right\rangle \sim\langle\boldsymbol{\Omega S} \boldsymbol{\Omega}\rangle$ and $\left\langle\operatorname{Tr}\left(\mathbf{S}^{2}\right)\right\rangle \sim\left\langle\boldsymbol{\Omega}^{2}\right\rangle$, it can be deduced that rotation also suppresses the strain production rate $\left\langle-\operatorname{Tr}\left(\mathbf{S}^{3}\right)\right\rangle /\left\langle\operatorname{Tr}\left(\mathbf{S}^{2}\right)\right\rangle^{3 / 2}$.

\section{CONCLUSION}

The structure of homogeneous rotating turbulence was investigated by measuring the instantaneous statistics of the scale-dependent perceived velocity gradient tensor supported by four fluid elements forming a regular tetrahedron. This method provides a multiscale analysis of the flow, in the physical space, and allows us to measure dynamical quantities which play a key role in turbulence, such as enstrophy and strain production. It also gives access to the relative orientations between the vorticity, strain eigenframe, vortex stretching vector, and rotation axis, thereby characterizing the structure of turbulence from a geometrical point of view. Four flows with similar $\operatorname{Re}_{\lambda}$ and different Rossby numbers were computed by direct numerical simulation. The two limits of nonrotating turbulence and turbulence expected to be affected by rotation at all scales $\left(\ell_{\mathrm{HZ}}<\eta\right)$ have been considered. The entire inertial range of scales was covered, from $r_{0} \lesssim \eta$ (representing the dissipative range) to $r_{0} \approx L / \sqrt{2}$.

The orientation of the flow with the rotating frame was investigated: Vorticity and the intermediate strain direction were found to be preferentially vertical, whereas the vortex stretching

\footnotetext{
${ }^{2}$ Strictly speaking, this quantity is rather a generalized enstrophy production rate [37], the standard one being defined as $\boldsymbol{\Omega} \mathbf{S} \boldsymbol{\Omega} / \boldsymbol{\Omega}^{2}$ [1].
} 
vector and strain eigenvector associated with the compression direction have a propensity to be horizontal. Although satisfied at any scale, these trends are the most stringent at $r_{0} \approx L / 3 \approx 1$. The flow visualizations displayed in Figs. 1(b)-1(d) show that this scale is the typical size of the large vortices visible in horizontal planes of rotating turbulence. The orientation of turbulence with the rotating frame is therefore, rather naturally, maximal at the scale of these structures. On a side note, the cyclone-anticyclone asymmetry was found to be maximal at the same characteristic scale $[19,37,43]$.

The other statistics show a continuous (and most often monotonic) evolution with scale. In particular, $\ell_{\mathrm{HZ}}$ (the Hopfinger-Zeman scale, at which inertia and rotation are comparable) and $\ell_{d}$ (scale at which rotation and dissipation balance) do not seem at first glance to influence these quantities. Such a result might be due to the fact that the inertial range is too narrow in the flows considered and would deserve to be tested in flows at higher Reynolds numbers. Nevertheless, a closer look at the data shows that most of the statistics obtained for run $4\left(\mathrm{Ro}^{(L)}=0.027\right)$ drastically differ from those of runs 2 and 3, despite the fact that the jump in $\operatorname{Ro}^{(L)}$ between runs 3 and 4 is identical to the one between runs 2 and 3. Recalling that run 4 is the only one for which $\ell_{\mathrm{HZ}}$ and $\ell_{d}$ are smaller than the Kolmogorov scale, the specificity of the statistics obtained in the corresponding flow might therefore be an indirect signature of these scales. Finally, all the statistics tend to Gaussian ones as $r_{0} \rightarrow L$ due to the decorrelation of velocities at the four tetrahedra vertices.

The statistics investigated in the present work reflect the Gaussianization of turbulence by rotation, a phenomenon that might be explained by the presence of inertial waves. Some of these statistics also illustrate the two-dimensionalization of turbulence by rotation. More precisely, rotation is shown to suppress the overall preferential orientation between vorticity and the strain eigenframe (except in "two-dimensional" regions of the flow where their relative statistical orientations are well defined) and the alignment between vorticity and vortex stretching vector. The latter effect induces a strong decrease of enstrophy production and strain production. The same conclusions hold at any scale of the flow. As already mentioned, a more refined characterization of the role of inertial waves and of the $2 \mathrm{D}$ regions of the flow in these statistics could be provided by performing the same analysis on a decomposed velocity field in which the contribution of the 3D fast manifold is separated from that of the 2D slow one [32].

Another interesting extension of this work would consist in investigating the Lagrangian statistics of the perceived velocity gradient tensor and of the associated deformation of the tetrahedra [12,13] in homogeneous rotating turbulence. The same tools could also be used to probe the structure of other anisotropic turbulent flows, such as those coupled to stratification or to a magnetic field.

\section{ACKNOWLEDGMENTS}

The author is grateful to C. Cambon, F. Godeferd, and A. Pumir for useful discussions and more specifically to F. Godeferd for providing the code. The simulations were performed using the numerical resources provided by GENCI-IDRIS and GENCI-CINES (Grant No. x20162a2206). This work was supported by the Agence Nationale pour la Recherche (Contract No. ANR-17-CE300003).

[1] A. Tsinober, An Informal Conceptual Introduction to Turbulence (Springer, Berlin, 2009).

[2] P. Vieillefosse, Local interaction between vorticity and shear in a perfect incompressible fluid, J. Phys. (Paris) 43, 837 (1982).

[3] B. J. Cantwell, Exact solution of a restricted Euler equation for the velocity gradient tensor, Phys. Fluids A 4, 782 (1992).

[4] C. Meneveau, Lagrangian dynamics and models of the velocity gradient tensor in turbulent flows, Annu. Rev. Fluid Mech. 43, 219 (2011). 
[5] A. Tsinober, E. Kit, and T. Dracos, Experimental investigation of the field of velocity gradients in turbulent flows, J. Fluid Mech. 242, 169 (1992).

[6] B. Galanti and A. Tsinober, Self-amplification of the field of velocity derivatives in quasi-isotropic turbulence, Phys. Fluids 12, 3097 (2000).

[7] M. Kholmyansky, A. Tsinober, and S. Yorish, Velocity derivatives in the atmospheric surface layer at $\operatorname{Re}_{\lambda}=10^{4}$, Phys. Fluids 13, 311 (2001).

[8] G. Gulitski, M. Kholmyansky, W. Kinzelbach, B. Lüthi, A. Tsinober, and S. Yorish, Velocity and temperature derivatives in high-Reynolds-number turbulent flows in the atmospheric surface layer. Part 1. Facilities, methods and some general results, J. Fluid Mech. 589, 57 (2007).

[9] W. T. Ashurst, A. R. Kerstein, R. M. Kerr, and C. H. Gibson, Alignment of vorticity and scalar gradient with strain rate in simulated Navier-Stokes turbulence, Phys. Fluids 30, 2343 (1987).

[10] M. Chertkov, A. Pumir, and B. I. Shraiman, Lagrangian tetrad dynamics and the phenomenology of turbulence, Phys. Fluids 11, 2394 (1999).

[11] A. Naso and A. Pumir, Scale dependence of the coarse-grained velocity derivative tensor structure in turbulence, Phys. Rev. E 72, 056318 (2005).

[12] H. Xu, A. Pumir, and E. Bodenschatz, The pirouette effect in turbulent flows, Nat. Phys. 7, 709 (2011).

[13] A. Pumir, E. Bodenschatz, and H. Xu, Tetrahedron deformation and alignment of perceived vorticity and strain in a turbulent flow, Phys. Fluids 25, 035101 (2013).

[14] A. Naso, A. Pumir, and M. Chertkov, Statistical geometry in homogeneous and isotropic turbulence, J. Turbul. 8, N39 (2007).

[15] A. Pumir and A. Naso, Statistical properties of the coarse-grained velocity gradient tensor in turbulence: Monte-Carlo simulations of the tetrad model, New J. Phys. 12, 123024 (2010).

[16] A. Pumir and A. Naso, Insight on turbulent flows from Lagrangian tetrads, C. R. Phys. 13, 889 (2012).

[17] A. Naso, A. Pumir, and M. Chertkov, Scale dependence of the coarse-grained velocity derivative tensor: Influence of large-scale shear on small-scale turbulence, J. Turbul. 7, N41 (2006).

[18] P. J. Staplehurst, P. A. Davidson, and S. B. Dalziel, Structure formation in homogeneous freely decaying rotating turbulence, J. Fluid Mech. 598, 81 (2008).

[19] F. Moisy, C. Morize, M. Rabaud, and J. Sommeria, Decay laws, anisotropy and cyclone-anticyclone asymmetry in decaying rotating turbulence, J. Fluid Mech. 666, 5 (2011).

[20] P. D. Mininni, D. Rosenberg, and A. Pouquet, Isotropization at small scales of rotating helically driven turbulence, J. Fluid Mech. 699, 263 (2012).

[21] A. Delache, C. Cambon, and F. Godeferd, Scale by scale anisotropy in freely decaying rotating turbulence, Phys. Fluids 26, 025104 (2014).

[22] L. Biferale, F. Bonaccorso, I. M. Mazzitelli, M. A. T. van Hinsberg, A. S. Lanotte, S. Musacchio, P. Perlekar, and F. Toschi, Coherent Structures and Extreme Events in Rotating Multiphase Turbulent Flows, Phys. Rev. X 6, 041036 (2016).

[23] F. S. Godeferd and F. Moisy, Structure and dynamics of rotating turbulence: a review of recent experimental and numerical results, Appl. Mech. Rev. 67, 030802 (2015).

[24] C. Cambon, N. N. Mansour, and F. S. Godeferd, Energy transfer in rotating turbulence, J. Fluid Mech. 337, 303 (1997).

[25] F. Waleffe, The nature of triad interactions in homogeneous turbulence, Phys. Fluids A 4, 350 (1992).

[26] S. Galtier, Weak inertial-wave turbulence theory, Phys. Rev. E 68, 015301 (2003).

[27] F. Bellet, F. S. Godeferd, J. F. Scott, and C. Cambon, Wave turbulence in rapidly rotating flows, J. Fluid Mech. 562, 83 (2006).

[28] A. Campagne, B. Gallet, F. Moisy, and P.-P. Cortet, Disentangling inertial waves from eddy turbulence in a forced rotating-turbulence experiment, Phys. Rev. E 91, 043016 (2015).

[29] Q. Chen, S. Chen, G. L. Eyink, and D. D. Holm, Resonant interactions in rotating homogeneous threedimensional turbulence, J. Fluid Mech. 542, 139 (2005).

[30] A. Sen, P. D. Mininni, D. Rosenberg, and A. Pouquet, Anisotropy and nonuniversality in scaling laws of the large-scale energy spectrum in rotating turbulence, Phys. Rev. E 86, 036319 (2012).

[31] A. Pouquet, R. Marino, P. D. Mininni, and D. Rosenberg, Dual constant-flux energy cascades to both large scales and small scales, Phys. Fluids 29, 111108 (2017). 
[32] M. Buzzicotti, H. Aluie, L. Biferale, and M. Linkmann, Energy transfer in turbulence under rotation, Phys. Rev. Fluids 3, 034802 (2018).

[33] M. Mory and E. J. Hopfinger, in Macroscopic Modelling of Turbulent Flows, edited by U. Frisch, J. B. Keller, G. C. Papanicolaou, and O. Pironneau, Lecture Notes in Physics Vol. 230 (Springer, Berlin, 1985), pp. 218-236.

[34] O. Zeman, A note on the spectra and decay of rotating homogeneous turbulence, Phys. Fluids 6, 3221 (1994).

[35] A. Naso and F. S. Godeferd, Statistics of the perceived velocity gradient tensor in a rotating turbulent flow, New J. Phys. 14, 125002 (2012).

[36] L. J. A. van Bokhoven, C. Cambon, L. Liechtenstein, F. S. Godeferd, and H. J. H. Clercx, Refined vorticity statistics of decaying rotating three-dimensional turbulence, J. Turbul. 9, N6 (2008).

[37] A. Naso, Cyclone-anticyclone asymmetry and alignment statistics in homogeneous rotating turbulence, Phys. Fluids 27, 035108 (2015).

[38] L. Del Castello and H. Clercx, Geometrical statistics of the vorticity vector and the strain rate tensor in rotating turbulence, J. Turbul. 14, 19 (2013).

[39] R. Rogallo, Numerical experiments in homogeneous turbulence, NASA Report No. 81315, 1981 (unpublished).

[40] A. Vincent and M. Meneguzzi, The spatial structure and statistical properties of homogeneous turbulence, J. Fluid Mech. 225, 1 (1991).

[41] A. Pumir, A numerical study of pressure fluctuations in three-dimensional, incompressible, homogeneous, isotropic turbulence, Phys. Fluids 6, 2071 (1994).

[42] D. Vallefuoco, A. Naso, and F. S. Godeferd, Small-scale anisotropy induced by spectral forcing and by rotation in non-helical and helical turbulence, J. Turbul. 19, 107 (2018).

[43] B. Gallet, A. Campagne, P.-P. Cortet, and F. Moisy, Scale-dependent cyclone-anticyclone asymmetry in a forced rotating turbulence experiment, Phys. Fluids 26, 035108 (2014).

[44] T. S. Lund and M. M. Rogers, An improved measure of strain state probability in turbulent flows, Phys. Fluids 6, 1838 (1994).

[45] J. Seiwert, C. Morize, and F. Moisy, On the decrease of intermittency in decaying rotating turbulence, Phys. Fluids 20, 071702 (2008).

[46] A. K. Kuczaj, B. J. Geurts, and D. D. Holm, Proceedings of the TSFP6 Conference, Seoul, 2009 (unpublished).

[47] B. J. Geurts, D. Holm, and A. Kuczaj, Proceedings of the IMS Turbulence Workshop, London, 2007 (unpublished), pp. 24-25, available at http://www3.imperial.ac.uk/pls/portallive/docs/1/18181703.PDF

[48] M. Kinzel, M. Wolf, M. Holzner, B. Lüthi, C. Tropea, and W. Kinzelbach, Simultaneous two-scale 3DPTV measurements in turbulence under the influence of system rotation, Exp. Fluids 51, 75 (2011). 